\title{
RESULTS OF DIFFERENT METHODOLOGIES IN THE TEACHING OF OCCUPATIONAL RISK PREVENTION FOR ADULTS IN VOCATIONAL TRAINING
}

\author{
Pasquale de Dato, Yolanda Hernández Navarro \\ Universitat Politècnica de València (SPAIN)
}

\begin{abstract}
Training in the prevention of occupational risks plays a very important role in prevention, especially if it is aimed at disadvantaged groups, who may compromise their access to the labor market in conditions of safety and psycho-physical comfort.

In the last years the implementation of Basic Occupational Risk Prevention Courses within the vocational training programs for work-linked employment (in Spain ETCOTE), which are aimed at these groups, has been made compulsory.

In this study, which gathers the conclusions of the first part of the research, some training experiences in ORP carried out in employment workshops are analyzed, evaluating their results in the medium term, and the new trends in training for adults are reviewed.

From the research carried out it becomes evident that a traditional or academic methodological approach (Direct Instruction) does not achieve an effective learning, obtaining better results in the long term in the cases of more practical methodologies, active and based on students' previous knowledge.

In the same way, positive results or at least a better learning environment, motivation and student participation are related to the use of gamification techniques in teaching.

The analytical methodology proposed is a first step towards a study, on a larger sample, of the results of training in occupational risk prevention that allows establishing, in a second phase, methodological guidelines for teachers of this training that take into account the profile of the students, the specialties of the professional certificates that are offered, the organization and management of the works and services that are offered in these types of programs.
\end{abstract}

Keywords: Didactic methodology analysis, ORP training, Adult learning, Teaching tools, Gamification.

\section{INTRODUCTION}

The objectives of the regulations of the training in occupational risk prevention are to improve working conditions and raise the level of protection of workers' safety and health [1]. For this reason, training plays a very important role in prevention after the identification and evaluation of occupational risks and especially if it is aimed at disadvantaged groups, at risk of social exclusion or far from the labour market, which, due to inexperience or acquired defects, may compromise their access and maintenance in the labour market in conditions of safety and psychophysical comfort.

As a result of the obligation to carry out Basic Occupational Risk Prevention courses as part of vocational training programs for employment in alternation with work (ETCOTE), it is extremely useful to establish methodological guidelines for this training, which take into account the profile of the participants, the specialties of the certificates of professionalism that are taught and the organization and management of the works and services offered in these types of programs to guide the promoting entities that carry them out.

The following article is the result of the research carried out for a final master's project and wants to provide an analytical methodology for the study of teaching results in Occupational Risk Prevention (ORP) from which to formulate, in other research, effective teaching guidelines for this type of training [2].

For this, the structures of the ETCOTE and their operation have been studied, the objectives established by the Ministry of Employment and Social Security in the modular risk prevention courses FCOS02 have been considered, some ORP learning experiences carried out in workshops have been analyzed, assessing their results in the medium term, and the new trends in training in general and for adults in particular have been reviewed. 


\subsection{Projects ETCOTE}

The ETCOTE projects, an acronym for School Workshop, House of Trades and Employment Workshop, can be defined as assistance programs that associate training for job promotion, the possibility of working for an entity, normally local administrations or supra-municipal.

Due to this healthcare nature, the beneficiaries of these programs are different disadvantaged groups that, due to age, education, social status or gender, find serious difficulties in entering the labour market. With few differences, all the projects are aimed exclusively at unemployed job seekers.

People with functional diversity, women victims of gender-based violence, people in or at risk of poverty, social exclusion or severe material deprivation, heads of single-parent families, young people under guardianship by the administration or subjects to the system of protection of minors, people over forty-five or fifty-five years (depending on the type of project), people with low qualifications and long-term unemployed are identified as "priority groups".

The training provided in the ETCOTE basically refers to the Professional Certificates related to the training specialties provided by the State Public Employment Service (SEPE). Training specialty is defined as the grouping of content, professional skills and technical specifications that respond to a set of work activities framed in a phase of the production process and with related functions, such as training specialties for General Use, Complementary Training and Modular Training.

The SEPE specifies that: "Complementary specialties all belong to the Professional Family of Complementary Training (FCO) and are considered transversal training in areas that are considered priorities both within the framework of the European Strategy for Employment and the National System Employment as in the guidelines established by the European Union. Priority areas are those related to information and communication technologies, the prevention of occupational hazards, environmental awareness, the promotion of equality, professional guidance and those others established by the competent administration."

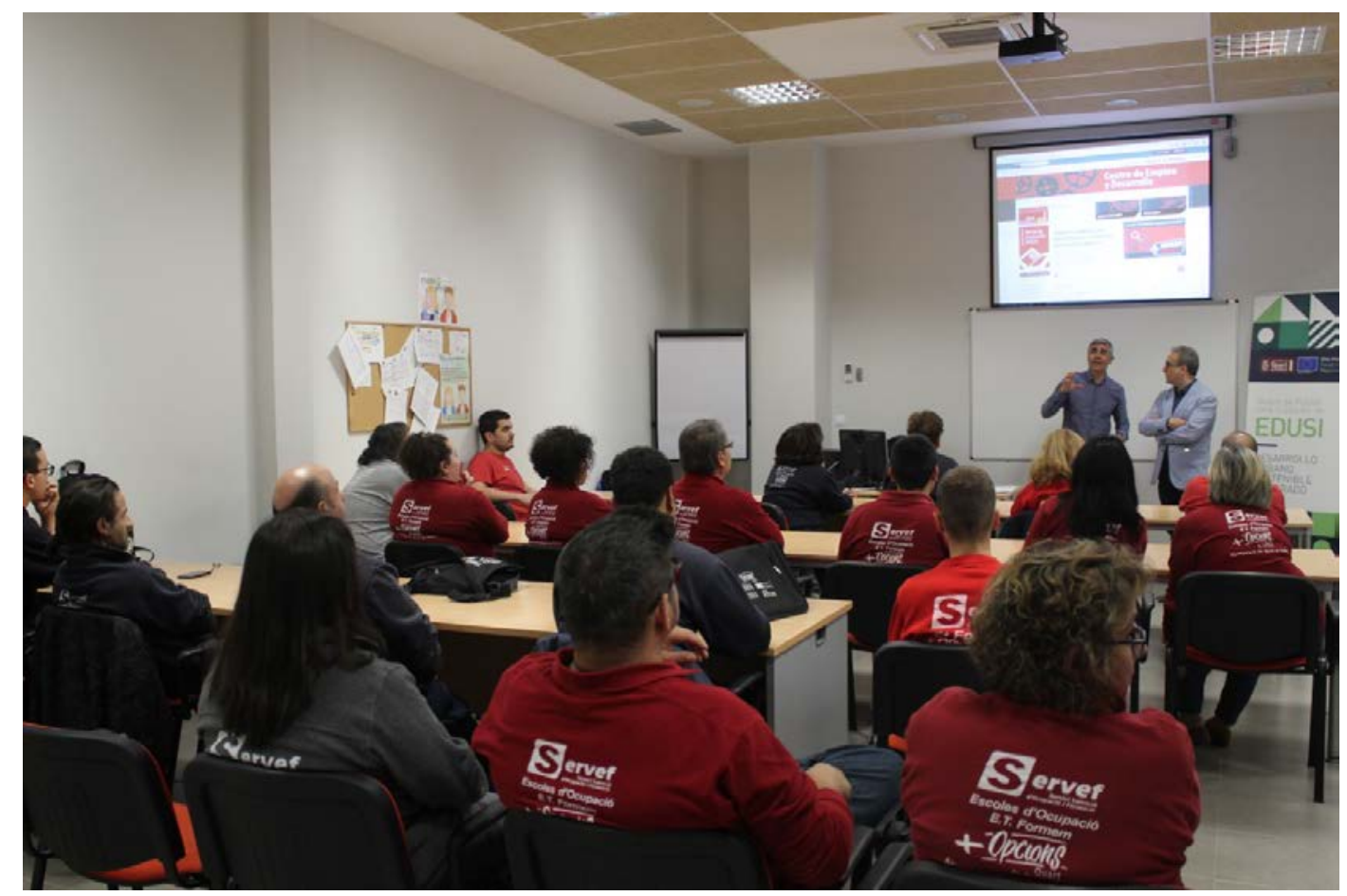

Figure 1: Student groups in the Quart1 and Quart2 groups.

\section{METHODOLOGY}

The research is based above all on an analysis of the teaching methodologies used in different training experiences in occupational risk prevention at ETCOTE; in evaluating the effectiveness of these methodologies to highlight the aspects to be improved and mark a route of good practices; in 
identifying the added value brought by the latest trends in teaching and new technologies to ORP training; as an analytical path of the results in this type of training.

For this, the specific bibliographic documentation has been consulted, the current regulations on preventive matters related to training have been studied, and the results have been tested in some real cases of Basic Training in Occupational Risk Prevention in the field of ETCOTE through short and long-term tests, comparison of the results obtained in the different evaluations to finally propose the most appropriate didactic strategies for training in ORP in ETCOTE projects.

\section{RESULTS}

\subsection{The reason for a complementary training in ORP}

The prevention of occupational risks is among the minimum mandatory contents that must be taught in the training aimed at obtaining a certificate of professional competences. Furthermore, as a type of modular training, it can be stated that in each of the training modules that constitute a certificate of professionalism, there are contents directly related to risk prevention and occupational health and safety. In addition, these are specific contents of the professional specialty of the certificate, that is, for each specialty, for each work activity or for each trade related to the certificate, there are exclusive and contextualized health and safety content of that specialty, activity or trade.

This is quite logical considering that the students in this training will enter the world of work with sufficient theoretical and practical preparation to fully develop their profession, including know-how in terms of job security. And if it is considered that the teacher qualified for the training the certificate should be a specialist in the sector with professional experience in that specific field, there is no doubt that no one better than that teacher could transmit the correct practices and knowledge of safety and health security of that area to students.

The reality, sadly, falls far behind this optimal scenario.

For some years now, in the orders and resolutions calling for the ETCOTE, a specific mention has been made of the compulsory nature of complementary training in the fields of gender equality, employability, the environment, digital skills and safety and health at work.

Its aim is to solve the problem of ensuring true quality training in occupational risk prevention.

\subsection{Study of actual ORP training cases}

In order to analyze actual training experiences in occupational risk prevention from which to extrapolate some considerations that could serve as a reference to establish methodological guidelines, three cases of basic training in ORP have been studied in the framework of three different projects of Workshop Schools in the province of Valencia that have involved six groups of 10 students each. To guarantee privacy and facilitate the study and research, the case studies have been identified with the generic geographical indications in "Quart 1", "Quart 2" and "Llíria 1", according to Table 1 and the students have been catalogued with a code that relates them to the project and its membership in the reference group with the structure of Table 2.

Table 1. Distribution of students in groups

\begin{tabular}{|l|c|c|c|}
\hline \hline \multicolumn{1}{|c|}{ Projects } & Groups & Initials number of students & Final number of students \\
\hline \multirow{4}{*}{ Quart 1 } & Group Q1.1 & 9 & 9 \\
\cline { 2 - 4 } & Group Q1.2 & 10 & 9 \\
\cline { 2 - 4 } & Group Q1.3 & 10 & 10 \\
\hline \multirow{3}{*}{ Quart 2 } & Group Q2.1 & 10 & 10 \\
\cline { 2 - 4 } & Group Q2.2 & 10 & 10 \\
\hline \multirow{2}{*}{ Lliria 1 } & Group L1.1 & 10 & 10 \\
\hline TOTALS & 6 & 59 & 58 \\
\hline \hline
\end{tabular}


Table 2. Structure of student identification code

\begin{tabular}{|c|c|c|}
\hline \hline Project & Group & Student \\
\hline Quart & $\mathbf{2}$ & $\mathbf{4}$ \\
\hline \multicolumn{3}{|c|}{ Student Q2.4 } \\
\hline
\end{tabular}

In the case of "Quart 1", occupational safety and health $(\mathrm{OSH})$ training has been given to three groups of 10 students each with a very elementary or no level of studies, each group with a different training specialty for a total of three Level I professional certificates.

The teacher responsible for training in OSH had previous non-training, but work experience, for more than 15 years as Head of Occupational Risk Prevention in a private company. This aspect seems to have been decisive in the definition of the teaching style oriented to meaningful learning that has transferred the business reality to the classroom. According to this perspective, it has been possible to transmit to the students that each concept treated in the training will be useful and applicable in their professional life, having adapted theory to practice [3].

The methodology used has been largely participatory with the aim of attracting the attention of students and keeping motivation alive. Likewise, the use of real cases from the professional experiences of the teacher and the contribution of previous experiences of the students has favoured a practical methodological approach.

About the resources used, it is worth highlighting presentations alternated with videos and practical exercises from which a more expository and traditional approach can be evidenced, and a series of visits in the workshop that have allowed to be put into practice the theoretical training in the real workplace [4].

However, the use of gamification of content to reinforce it and the continuous evaluation of learning through the KAHOOT application has represented an innovative factor in teaching that has positively marked the teaching-learning experience.

In the case of "Quart 2", however, OSH training has been given to two groups of 10 students each with respectively one and two different training specialties for a total of three Level II and Level III professional certificates. In the case of higher educational levels, the students had a higher school education, and even the majority, in the case of the Level III certificate, had a bachelor's or a degree.

The teacher in charge of the course has previous OSH training experience in private academies and a very short professional experience and different from the field of prevention. The contents have been taught in a pre-eminently theoretical way, following the programming of the ministerial document, although the participation of the students has been sought with the aim of attracting attention and keeping motivation alive.

On the educational resources, presentations and videos have been used exclusively in a clearly more expository and traditional approach.

In the case of "Llíria 1", the program has benefited a single group of 10 students who have completed a Level I certificate of professionalism. The training in $\mathrm{OSH}$, which has been given, has been carried out by the same teacher of the specialty of the certificate of professionalism. An interesting aspect, a priori, because it allows a more pertinent contextualization and an association of concepts adapted to the reality of the workplace.

On the other hand, the professor in charge of the course does not have previous experience in OSH training, this being his first experience and he has not worked in the field of prevention either. Furthermore, his work experience is specified only in the provision of non-regulated training.

Despite this professional specialization, the contents have been taught in a classical and preeminently theoretical way, but with constant reference to the specialty of the certificate.

Regarding to teaching resources, presentations and videos have been used exclusively with a clearly passive and expository method.

\subsubsection{Analysis of training results in case studies}

The case studies analysis has been based on the results obtained by the students in the different projects in relation to the methodologies used by the respective teachers. In order to maintain certain objectivity in the evaluation of the results, the notes of the final exam proposed by the different 
teachers have been taken into account and, to demonstrate the effectiveness of learning, the same students have been re-evaluated without prior notice and with the same examination one-year distance course delivery "Basic Occupational Risk Prevention".

The notes of the students regarding the first exam of the Module "FCOSO2_Básico de Prevención de Riesgos Laborales" and the same exam repeated one year later have been organized according to the qualifications established by Orden ESS/1897/2013, of October 10, by Real Decreto $34 / 2008$ is developed, in the ratings "Pass" (score from 5 to 6.9), "Merit" (from 7 to 8.9) and "Distinction" (from 9 to 10), obtaining percentages that have been useful to clarify possible correspondences between the level of previous studies of the students and the results of the exams, as shown in Table 3 for the first exam and in Table 4 for the repeated exam.

Table 3. Percentages of the ratings by groups in the first exam.

\begin{tabular}{|c|c|c|c|c|c|c|c|}
\hline & & Group Q1.1 & Group Q1.2 & Group Q1.3 & Group Q2.1 & Group Q2.2 & Group L1.1 \\
\hline \multirow{3}{*}{ 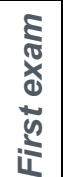 } & $\%$ Pass & $11 \%$ & $20 \%$ & $10 \%$ & $0 \%$ & $0 \%$ & $10 \%$ \\
\hline & $\%$ Merit & $33 \%$ & $70 \%$ & $40 \%$ & $0 \%$ & $60 \%$ & $60 \%$ \\
\hline & $\%$ Distinction & $56 \%$ & $10 \%$ & $50 \%$ & $100 \%$ & $40 \%$ & $30 \%$ \\
\hline
\end{tabular}

Table 4. Percentages of the ratings by groups in the repeated exam.

\begin{tabular}{|c|c|c|c|c|c|c|c|}
\hline & & Group Q1.1 & Group Q1.2 & Group Q1.3 & Group Q2.1 & Group Q2.2 & Group L1.1 \\
\hline \multirow{3}{*}{ 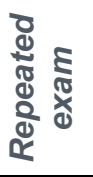 } & $\%$ Pass & $0 \%$ & $33,3 \%$ & $30 \%$ & $10 \%$ & $80 \%$ & $60 \%$ \\
\hline & $\%$ Merit & $55,5 \%$ & $33,3 \%$ & $60 \%$ & $70 \%$ & $20 \%$ & $30 \%$ \\
\hline & $\%$ Distinction & $44,5 \%$ & $33,3 \%$ & $10 \%$ & $20 \%$ & $0 \%$ & $10 \%$ \\
\hline
\end{tabular}

The average marks of each group, both for the first and the repeated exam, has shown interesting and significant behaviors of each group regardless of the level of studies of the students, which can be more directly related to the quality of the teaching-learning process or with the methodology. Table 5 shows, respectively, the average data obtained in the first exam, in the repeated exam one year away and the Figure 2 the comparisons between the two.

Table 5. Average data obtained in the first and in the repeated exam.

\begin{tabular}{|l|c|c|c|c|c|c|}
\hline \hline & Group Q1.1 & Group Q1.2 & Group Q1.3 & Group Q2.1 & Group Q2.2 & Group L1.1 \\
\hline Average 1 & 8,4 & 7,8 & 8,7 & 10 & 8,6 & 8,0 \\
\hline Average 2 & 8,3 & 7,7 & 7,2 & 8,1 & 6,0 & 6,6 \\
\hline
\end{tabular}

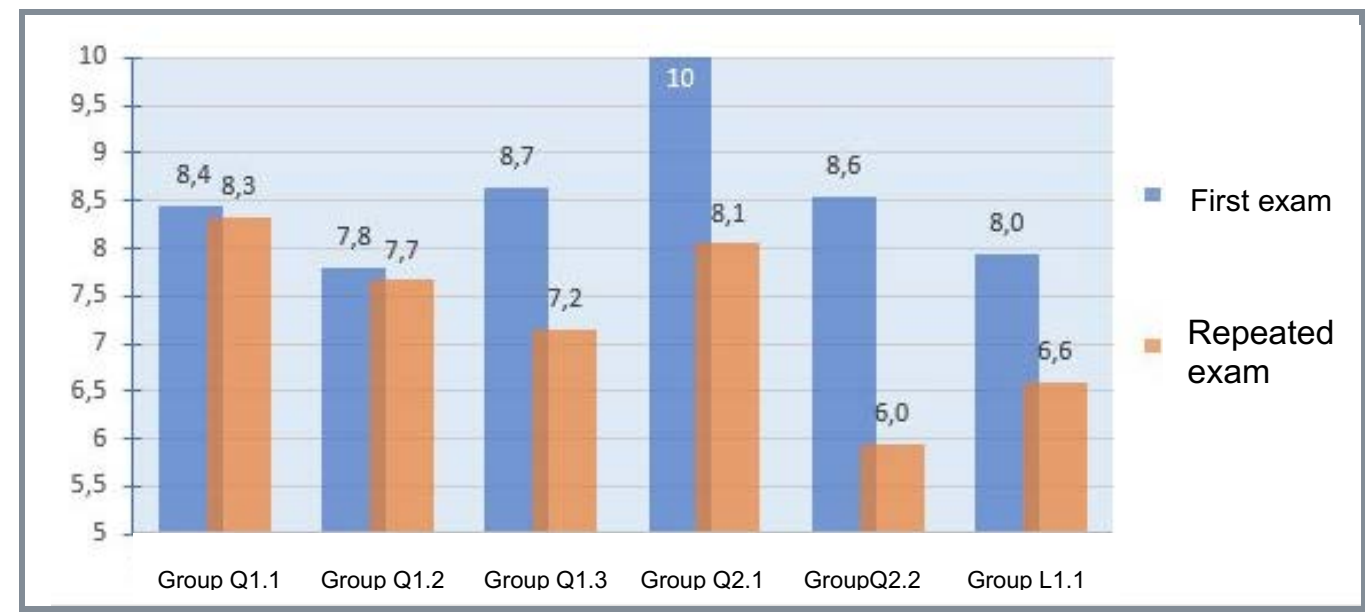

Figure 2. Comparison between averages of the results of the first and the repeated exams. 
Trends and patterns have been shown from the comparison of the results of the first exam with the results of the repeated exam, on which to make qualitative considerations of the methodology used.

As it can be seen in Figure 3, all the groups suffer a loss of acquired learning throughout a year, some in a limited way, others in a more accentuated way, marking a global average downward trend of $15 \%$.

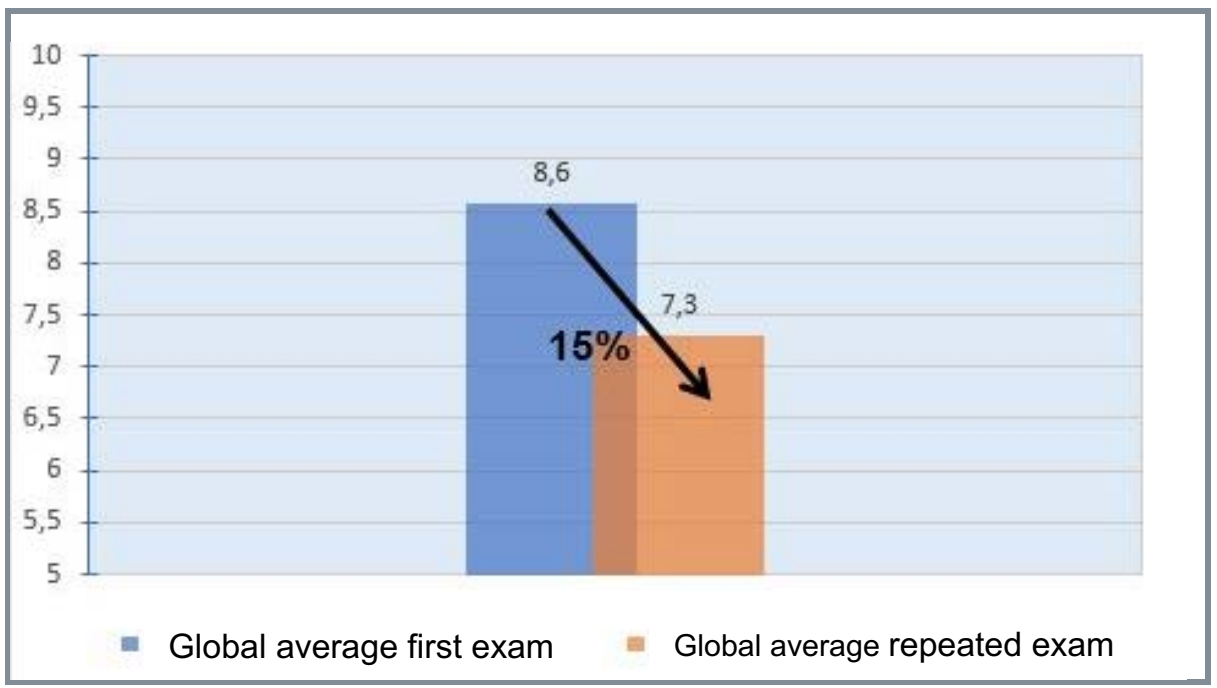

Figure 3. Overall trend of loss acquired learning in all groups.

This phenomenon forgetfulness is considered a normal aspect in the learning process due to the functioning of memory, since we can't preserve indefinitely in our memory everything we've learned is because we consciously forget information we don't make an effort to remember or, it seems to be the case, is because we forget information when it's not used or by "interference" that new information generates in the memory of older information or that old information makes it difficult to remember more recently acquired information.

However, this average is distributed differently in the various groups, as can be seen in the Figure 4 .

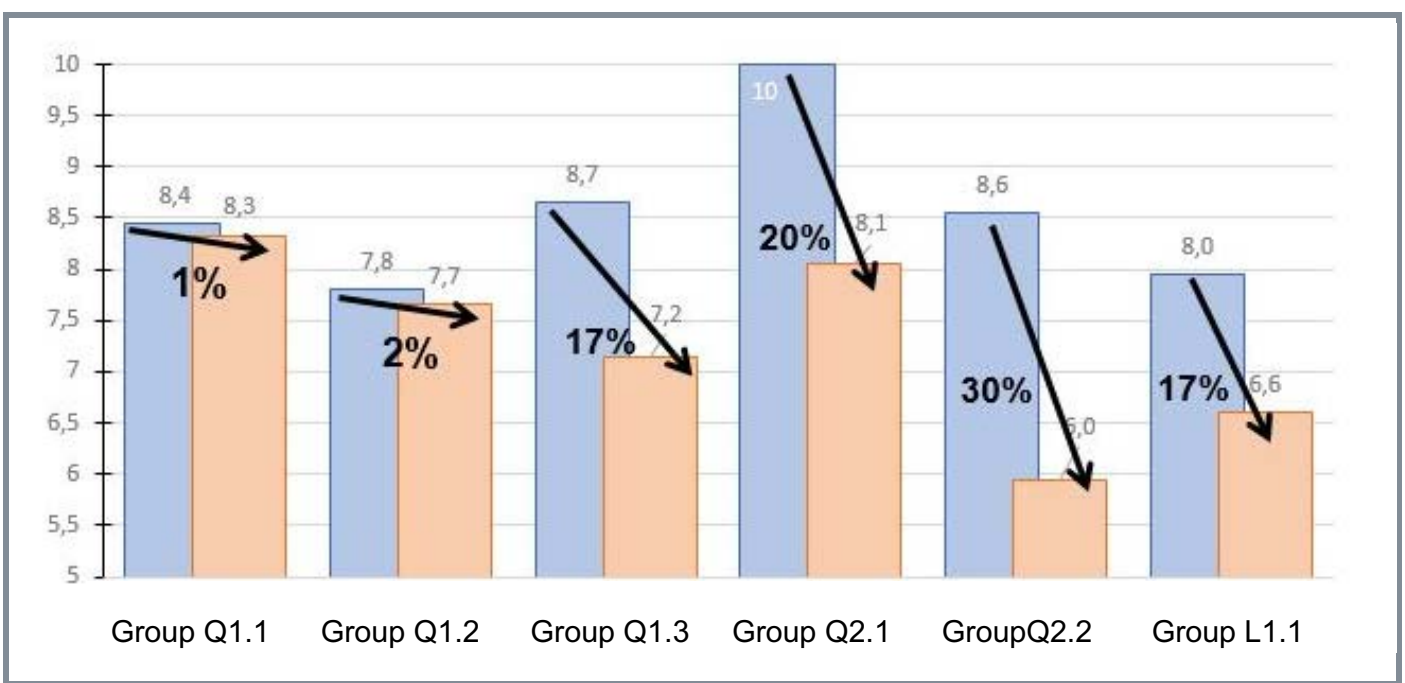

Figure 4. Analysis of content loss trends in the groups studied.

Analyzing the loss trends in each group can be evidenced how in the groups Q1.1 and Q1.2 the loss was minimal marking percentages respectively of $1 \%$ and $2 \%$, while in group Q1.3 and in group L1.1 there was a decrease to around the mean with values of $17 \%$. However, the groups Q2.1 and Q2.2, which suffered a decrease of $20 \%$ and $30 \%$ respectively, were surprising. 
This is a really interesting fact because the groups that register the least loss belong to the same teacher, as well as the groups that register the highest loss belong to other teachers with similar methodologies.

The only discordant data is that of Group Q1.3 which, even when referring to the teacher of Group Q1, nevertheless marks a trend similar to that of Group Q2.

This data could be justified considering the socio-cultural condition, performance or state of evolution of the group of students in Group Q1.3; but as data are difficult to prove or relate objectively, even if it is logical, it will be considered as a standard deviation.

The following conclusions have been extrapolated from the analysis of the results obtained by the students in the tests:

- The different level of study of the students has been reflected in the results of the exams. In particular, in the case of Group Q2.1, most of the students have a university degree or a Bachelor's degree prior to accessing the Professional Certificate and all of them have achieved the highest mark in the first exam, possibly not having adapted to the student's abilities. On the contrary, the same exam provided to the students of Group Q2.2 has resulted in a wider range of grades.

- All the groups have recorded, to a lesser or greater extent, a loss of knowledge acquired at a distance of one year of learning, being however, the groups related to a more practical methodology and meaningful learning, those that have suffered a minor loss.

- Contrary to expectations, the group with the highest level of studies has marked a significant drop $(20 \%)$ in the acquired learning, which may be explained by the lack of applicability of the concepts studied in the day-to-day work.

- Analyzing the evolution of the marks for each student has been found in some cases an improvement in learning despite the passage of time, in contrast to the rest of the students.

This could be explained by a direct relationship between the feedback of the exam and the work practice, when a student learns from his mistakes on the exam and/or correctly applies what he/she has learned in the day-to-day of his/her job.

\subsubsection{Analysis of the perception of training in ORP}

Generally, and in the case of the students and teachers interviewed in the study, almost all agree that the contents of a Basic Course on Occupational Risk Prevention are complex for those who have never treated the subject, monotonous, (some have even defined them tedious) but of general interest, although at a time far from the everyday reality.

In other words, safety at work continues to be conceived as a somewhat annoying obligation for the workers, although essential for their safety and that all obligations are complex and unattractive to them.

It is evident that there is a background communication problem in ORP training that results in a decrease in interest. In these cases a correct methodology could facilitate learning.

After interviewing the students about the training received, a general feeling of satisfaction, fun and learning new content was registered in groups Q.1, despite the difficulty of the contents. The response from the Q.2 groups was widespread satisfaction, although they considered the contents very useful but complex and boring. Finally, the interview with the students of group L.1 showed a general medium-low satisfaction mainly for the theoretical and normative contents, while the contextualization in the workplace was appreciated.

\section{CONCLUSIONS}

From the study of the six OSH training groups in ETCOTE, it has become evident that a traditional methodological approach does not achieve effective learning. Although it may seem that the shortterm results are excellent in the medium-long term, losses of information (on average 15\%) can be registered, either due to the heaviness of the information received (conscious forgetfulness) or due to the lack of connection between theoretical learning with day-to-day work practice (transferability).

However, better results (learning loss of 1-2\%) have been observed in long-term in the cases of more practical, active and knowledge-based methodologies (significant learning). Even occasionally 
improvements in results have been noted in relation to the short term, possibly due to the correction of errors through practice in the workplace.

Likewise, positive results or at least a better learning environment can be related in cases where gamification techniques have been used in teaching and attention, motivation and participation have been promoted through play [5].

The feeling of final satisfaction of the course has varied depending on the methodologies adopted in class.

It can also be underlined that despite the educational level, long-term learning tends to produce very similar content retention effects thresholds among students with different levels of education and training.

After a complete analysis and an exhaustive interpretation of the results and the experiences registered by the students, it has been possible to exclude as effective methods, for training in occupational risk prevention, those based on passive methodologies with a more traditional expository approach, by the evident need for a practical component that enables the transfer of concepts to real life. Likewise, the key factors for success in safety and health training in the workplace or at least the ETCOTE have been evidenced, such as applicability, participation, clarity of objectives, relevance, repetition, transfer and meaningful learning, to set some guidelines that should be taken into account when designing effective teaching programming for adults.

As seen in the most successful case studies analyzed, meaningful learning allows that, when the task to be learned can be related to the person's previous knowledge, learning is ensured. Hence the importance of finding out the background that the students have and relating them to the new ones who are going to work in the training situation [6].

The analysis, of course, has limitations dictated by the number of witnesses employed, by the previous educational differences in the student body, by the differences in the proposed exams, etc., but it may represent a first approximation for a system for an evaluation system of teaching quality in $\mathrm{OSH}$.

\section{REFERENCES}

[1] P. R.Gil-Monte, \& J. A. García-Juesas, "Otras actuaciones en materia de prevención." in Prevención de riesgos laborales. Instrumentos de aplicación. (I. Rosat Arced, M.C. Salcedo Beltrán, C. Alfonso Mellado, \& J.J. Agún González), 1532-1623, Valencia: Tirant lo Blanch, 2012.

[2] P. de Dato, "Directrices metodológicas para la formación en PRL en los ETCOTE", Master, Universidad Miguel Hernández, Elche, España, 2019.

[3] M. Almazán, Certificado de Profesionalidad en Docencia para la Formación, unpublished, 2017.

[4] O. Amat, Aprender a enseñar. Una visión práctica de la Formación de Formadores, Barcelona: Profit, 2009.

[5] P. Castillejo Poole, "Gamificación y prevención de riesgos laborales: Cuando la PRL es divertida", Prevencionistas, vol.26, 13-18, 2017 Retrieved from journal https://www.aepsal.com/wpcontent/uploads/2017/10/Prevencionistas-26.pdf

[6] I. Neciri, Hacia una didáctica general dinámica, Buenos Aires: Kapelusz, 1979. 